\title{
GAMMA-RAY BURSTS AND THE FIRST STARS
}

\author{
V. Bromm ${ }^{1}$
}

\begin{abstract}
Gamma-ray bursts (GRBs) triggered by the death of the first stars promise to provide a powerful probe into the state of the high-redshift universe. I will review the basic physics of how the first stars formed, evaluating whether they are suitable GRB progenitors. After providing estimates of the high-redshift GRB number density, I will discuss the utility of such bursts in probing the ionization state, and the degree of metal enrichment, of the early intergalactic medium. The prospects for these studies are bright, provided that we can fly a dedicated mission that combines a gamma-ray detector with on-board near-infrared capabilities.
\end{abstract}

\section{Introduction}

One of the key questions in modern cosmology is to understand how the first stars and galaxies ended the cosmic dark ages a few hundred million years after the Big Bang (Barkana \& Loeb 2001; Bromm et al. 2009; Loeb 2010). Prior to their emergence, the universe exhibited a simple state, devoid of complex structure, of any elements heavier than lithium, and of high-energy radiation fields. Within $\Lambda$ CDM cosmology, the first stars, the so-called Population III (Pop III), are predicted to form at $z \sim 20-30$ in dark matter minihalos of mass $\sim 10^{6} M_{\odot}$. The formation of the first bona-fide galaxies, implying the presence of long-lived stellar systems, may be delayed until more massive host halos become available (Bromm \& Yoshida 2011). Once the first sources of light have appeared on the cosmic scene, the universe was rapidly transformed through the input of ionizing radiation and heavy chemical elements. The character of this fundamental transition, as well as the assembly process of the first galaxies, crucially depended on the feedback exerted by Pop III stars (Ciardi \& Ferrara 2005). The feedback in turn is determined by the initial mass function (IMF) of the first stars (Bromm \& Larson 2004; Glover 2005). Although important uncertainties remain, the key prediction is that the Pop III IMF is biased towards high mass (top-heavy). At

${ }^{1}$ Department of Astronomy, University of Texas at Austin, USA

(C) EAS, EDP Sciences 2013 DOI: $10.1051 /$ eas/1361094 
least a fraction of the first stars could therefore have collapsed into massive black holes (BHs) at the end of their short lives, and thus provide viable gamma-ray burst (GRB) progenitors.

Upcoming facilities such as the James Webb Space Telescope (JWST), and the next generation of extremely-large telescopes on the ground (GMT, TMT, E-ELT) promise to open up a direct window into the first billion years of cosmic evolution (Gardner et al. 2006). Despite their exquisite sensitivity at near-IR wavelenghts, even these observatories may not be able to directly probe the first stars, unless they formed in massive clusters (Pawlik et al. 2011), or were gravitationally lensed (Rydberg et al. 2012). The only opportunity to probe individual Pop III stars may be to catch them at the moment of their explosive death. This could involve extremely energetic supernova (SN) events, such as hypernovae or pair-instability SNe (Hummel et al. 2012; Pan et al. 2012), or GRBs. The latter fate depends on whether Pop III stars could give rise to suitable collapsar progenitors, involving rapidly rotating massive stars (MacFadyen \& Woosley 1999). Since Pop III stars are predicted to fulfill both requirements (see the discussion below), GRBs are expected to be prevalent at very high redshifts. Indeed, GRBs may play a key role in elucidating primordial star formation, as well as the properties of the early intergalactic medium (IGM), given their extreme intrinsic brightness, both of the prompt $\gamma$-ray emission, as well as that of the prolonged afterglow.

A number of features render GRBs ideal probes of the epoch of first light (Loeb 2010): (i) Traditional sources to observe the high- $z$ universe, such as quasars and Lyman- $\alpha$ emitting galaxies, severely suffer from the effects of cosmological dimming, whereas GRB afterglows, if observed at a fixed time after the trigger, exhibit nearly-flat infrared fluxes out to very high $z$ (Ciardi \& Loeb 2000). This counterintuitive effect arises, because a fixed time interval in the observer frame translates into an increasingly early time in the source frame. Such earlier times in turn sample the rapidly decaying GRB lightcurve ${ }^{2}$ at the moment of maximal brightness, thus compensating for the cosmological dimming (increasing luminosity distance). (ii) In the hierarchical setting of cosmic structure formation, earlier times are dominated by lower-mass host systems. The massive hosts required for quasars and bright galaxies therefore are "dying out" at the highest redshifts (Mortlock et al. 2011). GRBs, on the other hand, mark the death of individual stars, which can form even in very low-mass systems. (iii) Finally, Pop III GRBs would provide very clean background sources to probe the early IGM. Again reflecting the low masses of their hosts, any proximity effect should be much reduced, as ionized bubbles are confined to the immediate vicinity of the Pop III system; the IGM would thus largely remain unperturbed. In addition, since GRB afterglow spectra can be described as featureless, broken power-laws (Vreeswijk et al. 2004), any signature imprinted by absorption and emission events along a given line of sight can be easily discerned. The outlook for GRB cosmology, therefore, is bright.

\footnotetext{
${ }^{2}$ Note that this argument pre-supposes that the power-law afterglow decay has been established, and may not be valid at the earliest times.
} 
Future missions, such as JANUS, Lobster, or SVOM, promise to fully unleash its potential.

\section{Formation of the first stars}

The longstanding consensus view has been that the conditions in the early universe favored the formation of predominantly massive stars, such that the Pop III IMF was top-heavy (Abel et al. 2002; Bromm et al. 2002; Bromm \& Larson 2004). This expectation rests on the much less efficient cooling in pure $\mathrm{H} / \mathrm{He}$ gas, where the only viable cooling agent is molecular hydrogen. The primordial gas can therefore reach temperatures of only $\sim 200 \mathrm{~K}$, compared to the $10 \mathrm{~K}$ reached in dust-cooled molecular clouds in the present-day Milky Way. The correspondingly enhanced thermal pressure is reflected in a Jeans mass that is larger by one to two orders of magnitude in the Pop III case. Another element of this "standard model" of primordial star formation has been that the first stars formed typically in isolation, one per minihalo.

Recently, beginning with work done in 2009, this traditional paradigm has been refined in important ways (Turk et al. 2009; Stacy et al. 2010; Clark et al. 2011; Greif et al. 2011, 2012). Supercomputing power, as well as algorithmic advances, now enable us to follow the protostellar collapse to densities, $n \sim 10^{22} \mathrm{~cm}^{-3}$, where the initial hydrostatic core forms in the center of the cloud (Yoshida et al. 2008). Crucially, the computations can now also be extended into the main accretion phase. An important lesson has been that accretion is mediated through a nearKeplerian disk, similar to present-day star formation. The hot conditions in the surrounding cloud result in extremely large rates of infall onto the disk $\left(\dot{M} \propto T^{3 / 2}\right)$; this rapid mass loading drives the disk inevitably towards gravitational instability, such that a small multiple of Pop III protostars emerges, often dominated by a binary system. It is not yet possible to extend such ab-initio simulations all the way to the completion of the protostellar assembly process; the final mass of Pop III stars and their final IMF are thus still subject to considerable uncertainty. However, first attempts to carry out the radiation-hydrodynamical calculations required to treat the late accretion phase, where protostellar feedback tends to limit further infall, have confirmed the basic prediction: the first stars were typically massive, with masses of a few $\sim 10 M_{\odot}$, although rarely very massive $\left(>100 M_{\odot}\right)$, as previously thought, forming as a member of small multiple systems (McKee \& Tan 2008; Hosokawa et al. 2011; Stacy et al. 2012a).

\subsection{Collapsar requirements}

Are Pop III stars suitable GRB progenitors? To successfully trigger a collapsar event, the leading contender for long-duration GRBs (Woosley 1993; MacFadyen et al. 2001), a number of conditions have to be met. These are quite stringent, and often difficult to fulfill simultaneously (Zhang \& Fryer 2004; Petrovic et al. 2005; Belczynski et al. 2007).

The first requirement for a collapsar central GRB engine, the emergence of BH remnants, is thus fulfilled. The binary nature of Pop III stars may also enable 


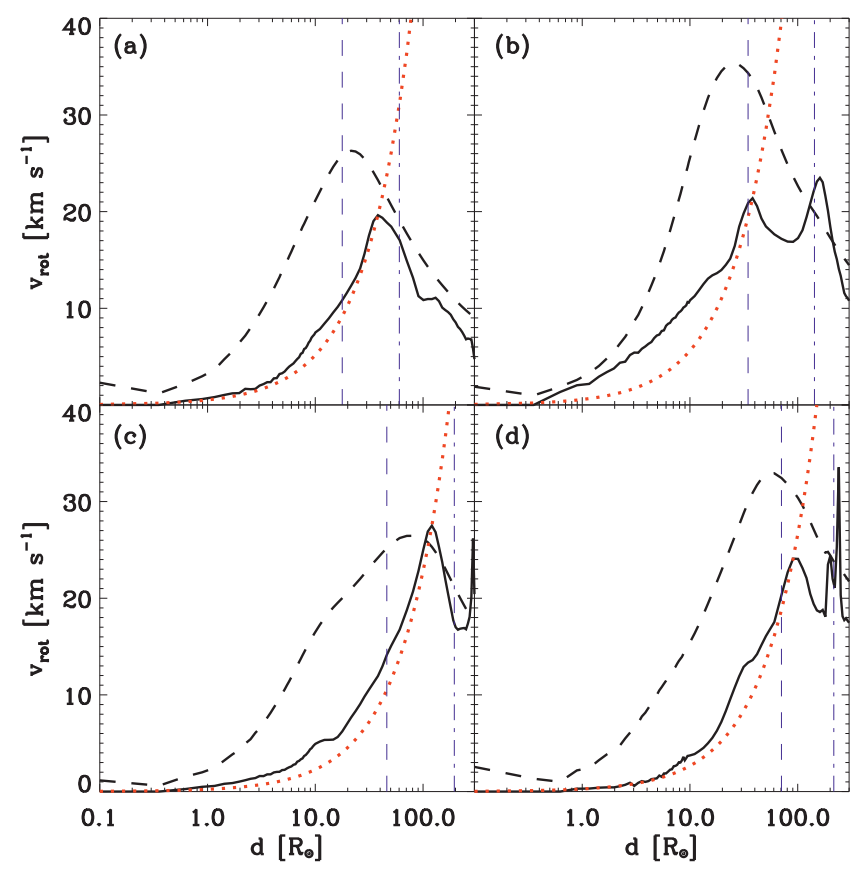

Fig. 1. Rotational velocities in Pop III protostars (from Stacy et al. 2012b). The panels show the situation in four statistically independent minihalos. Solid lines: the rotational velocity as a function of radial distance from the protostellar center. Dashed lines: Keplerian velocity vs. radius. Dotted lines: rotational velocity expected for solidbody rotation. In each panel the vertical lines mark the location of the protostellar "surface", defined as the boundary of the hydrostatic core (dashed), or the radius of the photosphere (dot-dashed). It is evident that rotational velocities comprise sizable fractions of the maximal, Keplerian, values. The caveat here is that the simulations could follow the evolution only for $\sim 10 \mathrm{yr}$ after the formation of the initial hydrostatic core.

them, if the binary is sufficiently close to allow for Roche-lobe overflow and a common-envelope phase, to expel the extended hydrogen (and helium?) envelope. This may be crucial to prevent the quenching of the relativistic jet, launched by the central engine (Bromm \& Loeb 2006; but see Suwa \& Ioka 2011). What about the additional requirement that the collapsar progenitor retains enough angular momentum? This question ties in with the rate of rotation of Pop III stars, where almost nothing is known yet. A first attempt to address this within a fully cosmological context has recently been carried out (Stacy et al. 2011, 2012b), indicating that the first stars may have typically been very fast rotators, with surface rotation speeds of a few $10 \%$ of the break-up value (see Fig. 1). Such high rates of rotation would have important consequences for Pop III stellar evolution, possibly enabling strong mixing currents, and for the fate encountered at death 
(Yoon et al. 2006). Thus, it is plausible that all requirements for a collapsar central engine were in place in the early universe. The next question now is: How common were Pop III GRBs, and do current or planned missions have a fair chance to detect them?

\subsection{How frequent were Pop III bursts?}

Briefly after the cosmological distance scale to GRBs had been established, it was realized that they provide a powerful probe of the cosmic star formation history, extending out to very high redshifts where the first stars are expected to form (Lamb \& Reichart 2000; Bromm \& Loeb 2002). As a case in point, we now have examples of such bursts at very high redshifts, with the spectroscopically confirmed GRB 090423 at $z \simeq 8.2$ (Salvaterra et al. 2009; Tanvir et al. 2009), and a photometrically constrained candidate at $z \sim 9.4$ (Cucchiara et al. 2011). In addition, the radio afterglow of GRB 090423 has been detected with the VLA (Chandra et al. 2010), providing useful constraints on the afterglow energetics and geometry, as well as on the circumburst density. From these observations, we have learned that the afterglow properties of the very high- $z$ bursts are not significantly different from the more local sample.

To explore the likely space of discovery, it is important to construct models of the high-redshift GRB rate. Schematically, this involves the following framework (for details, see Bromm \& Loeb 2006):

$$
\frac{d N_{\mathrm{GRB}}^{\mathrm{obs}}}{d z}=\psi_{\mathrm{GRB}}^{\mathrm{obs}}(z) \frac{\Delta t_{\mathrm{obs}}}{(1+z)} \frac{d V}{d z},
$$

where $d N_{\text {GRB }}^{\text {obs }}$ is the number of GRBs, as observed with a given instrument, from within a redshift interval $d z, \psi_{\mathrm{GRB}}^{\mathrm{obs}}$ the number of bursts per comoving volume, and the other symbols have their usual meaning. The connection between the burst number density and cosmic star formation rate density (SFRD) can be expressed via:

$$
\psi_{\mathrm{GRB}}^{\mathrm{obs}}(z)=\eta_{\mathrm{GRB}} \psi_{*}(z) \int_{L_{\lim }(z)}^{\infty} p(L) d L,
$$

where $\psi_{*}(z)$ is the cosmic SFRD, $\eta_{\text {GRB }}$ the GRB formation efficiency, $p(L)$ the GRB luminosity function, and $L_{\lim }(z)$ the minimum intrinsic luminosity required to detect the burst with a given instrument, from a given redshift.

Most of the intricacies come in when dealing with the efficiency factor. For simplicity, one could assign a constant value, possibly calibrating it to the observed Pop I/II value: $\eta_{\text {GRB }} \sim 10^{-9}$ bursts per unit solar mass (Bromm \& Loeb 2006). Within such an idealized model, one typically estimates that of order $10 \%$ of all Swift GRBs should originate from $z>5$, with of order 0.1 Pop III bursts per year. Detection of a Pop III burst may thus lie just outside of the Swift capabilities, unless we get lucky. However, the real situation is likely much more complicated. The GRB efficiency could well depend on redshift, or on environmental factors, such as the metallicity of the host system (Langer \& Norman 2006). Since the early modeling of the GRB redshift distribution, significant refinements have been 
added (Daigne et al. 2006; Campisi et al. 2011; deSouza et al. 2011; Ishida et al. 2011; Elliott et al. 2012). It is important, though, to not lose sight of the inherently very uncertain nature of this enterprise.

Among the most crucial uncertainties is the physical mechanism responsible for terminating the early mode of predominantly massive Pop III stars. Current thinking often posits that this Pop III to II transition is brought about by chemical feedback. The idea is that the cooling ability of star forming gas is greatly enhanced once it has been enriched with the first heavy elements beyond a threshold level, termed the "critical metallicity" (of order $Z_{\text {crit }} \sim 10^{-4} Z_{\odot}$ ). The underlying physics is complex. Some models claim that fine-structure lines of neutral oxygen and singly-ionized carbon may drive this transition (Bromm \& Loeb 2003); others identify dust cooling as the key agent (Schneider et al. 2006). If dust were indeed responsible, predicted values of $Z_{\text {crit }}$ are typically smaller by one to two orders of magnitude, compared with the fine-structure scenarios.

\section{GRBs as probes of the early IGM}

Assuming standard, shocked-synchrotron theory, the properties of Pop III afterglows have been worked out (Gou et al. 2004; Inoue et al. 2007). Consistently, across a wide range of wavelengths, from the near-IR to radio, as well as in the X-ray bands, flux levels are predicted that bring such Pop III bursts within reach of existing and planned instruments. A key uncertainty in such modeling is what to assume for the circumburst density (Wang et al. 2012). If we can identify these bursts through rapid follow-up in the near-IR, they will provide us with exquisite background sources to probe the early IGM. Firstly, we can place constraints on the ionized fraction of the high- $z$ IGM, as a function of redshift. This would provide a much more discerning picture of the cosmic reionization history, compared to the integral constraint from WMAP. In the latter case, by measuring the optical depth to Thomson scattering along the travel path of a CMB photon from the surface of last scattering to $z=0$, we cannot distinguish between models that can be quite different, but happen to yield the same line-of-sight integral. The basic idea is to exploit the absorption strength in the red damping wing of the Lyman- $\alpha$ resonance, which is very sensitive to any residual IGM neutral fraction (Barkana \& Loeb 2004). This idea has been tested with the exquisite spectrum taken for GRB 050904 at $z \simeq 6.3$ (Totani et al. 2006). The problem there proved to be the strong local column in neutral hydrogen, which completely overwhelmed any contribution from the general IGM. Again, the hope is that if we go to Pop III bursts, such local contamination would not be a problem, given that the first stars are exptected to form in low-mass host systems. Any local damping would then be small compared with the comological signal.

A second use of a Pop III GRB background source is to scrutinize the degree and nature of metal enrichment in the pre-galactic universe (see Fig. 2). The first stars are predicted to form in a highly biased region of the Gaussian random field of density fluctuations, such that their formation sites are strongly clustered. Any Pop III burst would then likely explode in a region that already may have been 

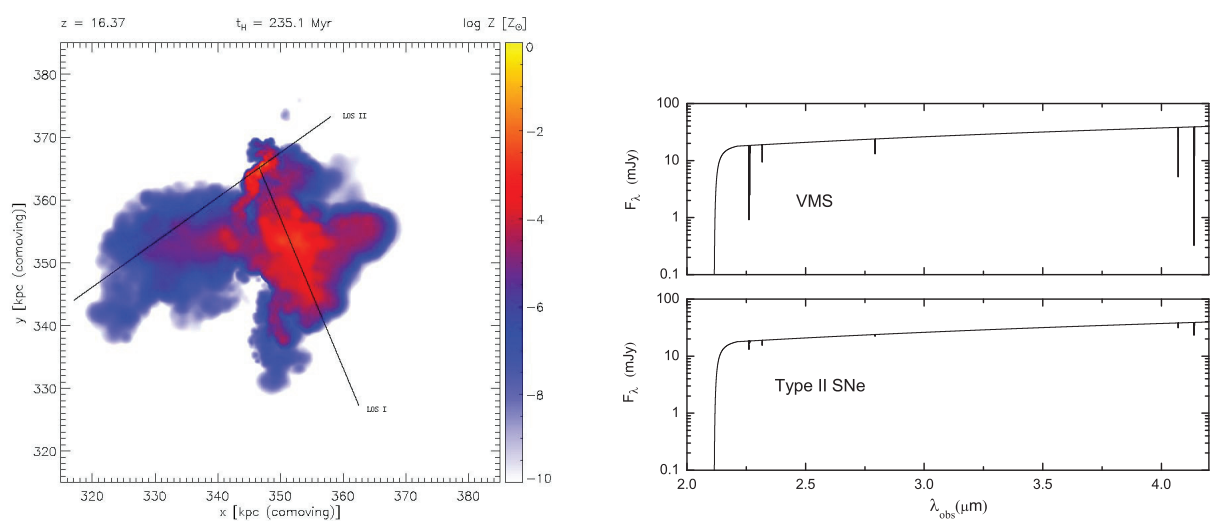

Fig. 2. Probing pre-galactic metal enrichment (from Wang et al. 2012). Left panel: metallicity distribution in a region of clustered Pop III star formation. The box size is $70 \mathrm{kpc}$ (comoving), shown at $z \simeq 16.4$. A Pop III GRB explodes at the intersection of the two lines. Right panel: resulting near-IR absorption spectra. We consider two different cases for the Pop III SN enrichment, from conventional core-collapse (Type II) and from a very massive star (VMS) progenitor. The situation corresponds to 1 day (in the observer frame) after the GRB trigger. The lines are imprinted by low-ionization species of $\mathrm{C}, \mathrm{O}, \mathrm{Si}$, and Fe. The sharp cutoff at lower wavelengths is due to the complete absorption of Ly $\alpha$ radiation in the neutral IGM. The VMS absorption-line signal should well be within the capabilities of the JWST.

enriched by a small number of SNe (Greif et al. 2010). The diagnostic provided by a high signal-to-noise, near-IR spectrum of a Pop III afterglow may allow us to not only measure the overall metallicity at a given redshift. Additionally, we may also be able to distinguish between the abundance pattern from different kinds of explosion, such as a pair-instability SN, a hypernova, or a more conventional corecollapse (Type II) event (Karlsson et al. 2013). A lower-redshift, $z \simeq 2$, example of this diagnostic is GRB 081008, where high-resolution spectroscopy with the Very Large Telescope has probed the host interstellar medium (D'Elia et al. 2011).

\section{Signature of Pop III bursts}

An important unsolved problem in GRB cosmology is how to uniquely identify possible Pop III bursts. High-redshift in itself is not sufficient, because different stellar populations will form contemporaneously, at least at $z \leq 15$. Attempts have been made to work out signatures that rely entirely on the gamma-ray emission, basically derived from the higher BH masses expected for Pop III remnants (e.g., Mészáros \& Rees 2010). However, such diagnostics appear very uncertain, not least because: How would we test or calibrate such gamma-ray-only markers? The commonly held notion that Pop III bursts could be unambiguously identified via the absence of any metal absorption lines in their afterglow spectra may not 
work either (see the argument in the previous section). What are we then left with? The metal-bubbles discussed above would originate at distances exceeding a few (physical) kpc from the burst. The immediate "near-zone" of the Pop III GRB, however, would still be chemically pristine. A unique identifier for Pop III bursts may thus be an ensemble of $\mathrm{H} / \mathrm{He}$ emission lines, possibly on top of the metal absorption signal originating farther away from the burst. The emission lines would arise as recombination radiation in the compact H II region powered by the UV-ionizing flux from the GRB afterglow. Such an emission line signature still needs to be worked out in detail, to see whether the line fluxes are sufficiently bright to render them detectable.

\section{References}

Abel, T., Bryan, G.L., \& Norman, M.L., 2002, Science, 295, 93

Barkana, R., \& Loeb, A., 2001, Phys. Rep., 349, 125

Barkana, R., \& Loeb, A., 2004, ApJ, 601, 64

Belczynski, K., Bulik, T., Heger, A., \& Fryer, C.L., 2007, ApJ, 664, 986

Bromm, V., Coppi, P.S., \& Larson, R.B., 2002, 564, 23

Bromm, V., \& Larson, R.B., 2004, ARA\&A, 42, 79

Bromm, V., \& Loeb, A., 2002, ApJ, 575, 111

Bromm, V., \& Loeb, A., 2003, Nature, 425, 812

Bromm, V., \& Loeb, A., 2006, ApJ, 642, 382

Bromm, V., Yoshida, N., Hernquist, L., \& McKee, C.F., 2009, Nature, 459, 49

Bromm, V., \& Yoshida, N., 2011, ARA\&A, 49, 373

Campisi, M.A., Maio, U., Salvaterra, R., \& Ciardi, B., 2011, MNRAS, 416, 2760

Chandra, P., Frail, D.A., Fox, D., et al., 2010, ApJ, 712, L31

Ciardi, B., \& Ferrara, A., 2005, Space Sci. Rev., 116, 625

Ciardi, B., \& Loeb, A., 2000, ApJ, 540, 687

Clark, P.C., Glover, S.C.O., Smith, R.J., et al., 2011, Science, 331, 1040

Cucchiara, A., Cenko, S.B., Bloom, J.S., et al., 2011, ApJ, 736, 7

Daigne, F., Rossi, E.M., \& Mochkovitch, R., 2006, MNRAS, 372, 1034

D'Elia, V., Campana, S., Covino, S., et al., 2011, MNRAS, 418, 680

de Souza, R.S., Yoshida, N., \& Ioka, K., 2011, A\&A, 533, A32

Elliott, J., Greiner, J., Khochfar, S., et al., 2012, A\&A, 539, A113

Gardner, J.P., Mather, J.C., Clampin, M., et al., 2006, Space Sci. Rev., 123, 485

Glover, S.C.O., 2005, Space Sci. Rev., 117, 445

Gou, L.J., Mészáros, P., Abel, T., \& Zhang, B., 2004, ApJ, 604, 508

Greif, T.H., Glover, S.C.O., Bromm, V., \& Klessen, R.S., 2010, MNRAS, 387, 1021

Greif, T.H., Springel, V., White, S.D.M., et al., 2011, ApJ, 737, 75

Greif, T.H., Bromm, V., Clark, P.C., et al., 2012, MNRAS, 424, 399

Hosokawa, T., Omukai, K., Yoshida, N., \& Yorke, H.W., 2011, Science, 334, 1250

Hummel, J.A., Pawlik, A.H., Milosavljević, M., \& Bromm, V., 2012, ApJ, 755, 72

Inoue, S., Omukai, K., \& Ciardi, B., 2007, MNRAS, 380, 1715 
Ishida, E.E.O., de Souza, R.S., \& Ferrara, A., 2010, MNRAS, 418, 500

Karlsson, T., Bromm, V., \& Bland-Hawthorn, J., 2013, Rev. Mod. Phys., in press [arXiv:1101.4024]

Lamb, D.Q., \& Reichart, D.E., 2000, ApJ, 536, 1

Langer, N., \& Norman, C.A., 2006, ApJ, 638, L63

Loeb, A., 2010, "How did the first stars and galaxies form?" (Princeton Univ. Press: Princeton)

MacFadyen, A.I., \& Woosley, S.E., 1999, ApJ, 524, 262

MacFadyen, A.I., Woosley, S.E., \& Heger, A., 2001, ApJ, 550, 410

McKee, C.F., \& Tan, J.C., 2008, ApJ, 681, 771

Mészáros, P., \& Rees, M.J., 2010, ApJ, 715, 967

Mortlock, D.J., Warren, S.J., Venemans, B., et al., 2011, Nature, 474, 616

Pan, T., Kasen, D., \& Loeb, A., 2012, MNRAS, 422, 2701

Pawlik, A.H., Milosavljević, M., \& Bromm, V., 2011, ApJ, 731, 54

Petrovic, J., Langer, N., Yoon, S.-C., \& Heger, A., 2005, A\&A, 435, 247

Rydberg, C.-E., Zackrisson, E., Lundqvist, P., \& Scott, P., 2012, MNRAS, submitted [arXiv: 1206.0007]

Salvaterra, R., Della Valle, M., Campana, S., et al., 2009, Nature, 461, 1258

Schneider, R., Omukai, K., Inoue, A.K., \& Ferrara, A., 2006, MNRAS, 369, 1437

Stacy, A., Greif, T.H., \& Bromm, V., 2010, MNRAS, 403, 45

Stacy, A., Bromm, V., \& Loeb, A., 2011, MNRAS, 413, 543

Stacy, A., Greif, T.H., \& Bromm, V., 2012a, MNRAS, 422, 290

Stacy, A., Greif, T.H., Klessen, R.S., Broom, V., \& Loeb, A., 2012b, MNRAS, submitted [arXiv: 1209.1439]

Suwa, Y., \& Ioka, K., 2011, ApJ, 726, 107

Tanvir, N.R., Fox, D.B., Levan A.J., et al., 2009, Nature, 461, 1254

Totani, T., Kawai, N., Kosugi, G., et al., 2006, PASJ, 58, 485

Turk, M.J., Abel, T., \& O'Shea, B.W., 2009, Science, 325, 601

Vreeswijk, P.M., Ellison, S.L., Ledoux, C., et al., 2004, A\&A, 419, 927

Wang, F.Y., Bromm, V., Greif, T.H., et al., 2012, ApJ, 760, 27

Woosley, S.E., 1993, ApJ, 405, 273

Yoon, S.-C., Langer, N., \& Norman, C., 2006, A\&A, 460, 199

Yoshida, N., Omukai, K., \& Hernquist, L., 2008, Science, 321, 669

Zhang, W., \& Fryer, C.L., 2004, in "Stellar Collapse", ed. C.L. Fryer (Dordrecht: Kluwer), 327 
